\title{
Diagnóstico do defeito ósseo de Stafne por meio de tomografia computadorizada de feixe cônico: relato de caso
}

\section{Diagnosis of Stafne's bone defect with cone beam computed tomography: Case Report}

\author{
Eduardo Murad Villoria \\ Mestrando em Clínicas Odontológicas - ênfase em Radiologia \\ Odontológica e Imaginologia da PUC-MINAS. \\ Christian Hellen Rodrigues da Cunha \\ Mestrando em Clínicas Odontológicas - ênfase em Radiologia \\ Odontológica e Imaginologia da PUC-MINAS. \\ LuCIANo Andrei Francio \\ Mestrando em Clínicas Odontológicas - ênfase em Radiologia \\ Odontológica e Imaginologia da PUC-MINAS. \\ JULIANA NoIA \\ Especialista em Radiologia Oral pela Universidade Gama Filho. \\ Paulo Taitson \\ Professor do curso de Mestrado Acadêmico \\ em Clínicas Odontológicas da PUC-MINAS. \\ KYRIA SPYRo SPYRIDES \\ Professora do Curso de Odontologia da Universidade Gama Filho. \\ FLÁVIO RICARDO MANZI \\ Coordenador do Curso de Mestrado Acadêmico em Clínicas Odontológicas \\ - ênfase em Radiologia Odontológica e Imaginologia da PUC-MINAS.
}

\section{RESUMO}

O defeito ósseo de Stafne possui o diagnóstico controverso por apresentar imagem semelhante às lesões benignas e malignas dos maxilares, principalmente quando é visualizado em radiografias convencionais, o que pode proporcionar intervenções cirúrgicas desnecessárias. O presente trabalho tem como objetivo descrever um relato de caso clínico de defeito ósseo de Stafne diagnosticado por meio de tomografia computadorizada de feixe cônico (TCFC), demonstrando as características imaginológicas no exame tomográfico.

Palavras-chave: DIAGNÓSTICO, ANORMALIDADES MAXILOMANDIBULARES, TOMOGRAFIA COMPUTADORIZADA DE FEIXE CÔNICO.

\begin{abstract}
The Stafne's bone defect have controversial diagnoses because it can be mistaken with benign and malignant lesions of the jaws, especially when observed in conventional radiographs that can take to unnecessary surgical interventions. This paper aims to describe a clinical case of a Stafne's bone defect diagnosed with cone beam computed tomography $(\mathrm{CBCT})$ showing the tomographic imaging features

Keywords: DIAGNOSIS, MAXILLOMANDIBULAR ABNORMALITIES, CONE BEAM COMPUTED TOMOGRAPHY
\end{abstract}




\section{INTRODUÇÃO}

O defeito ósseo de Stafne foi descrito pela primeira vez por Edward Stafne em 1942, que relatou 35 casos de imagens radiolúcidas, uniloculares, arredondadas ou ovaladas, bem circunscritas e localizadas abaixo do canal mandibular, entre o primeiro molar inferior e o ângulo da mandíbula1,2,3. Esta condição é frequentemente ocasionada pela inclusão de tecido mole na estrutura óssea adjacente ${ }^{4}$. 0 tecido da glândula salivar submandibular tem sido o achado histológico mais comum e, em poucos casos, músculos, vasos sanguíneos, gordura, tecido fibroso ou tecido linfoide têm sido relatados ${ }^{5}$.

A etiologia do defeito ósseo de Stafne não é bem definida. Muitos autores aceitam a hipótese de que a causa tem relação congênita ${ }^{1,6,7,8}$, no entanto outros estudiosos afirmam que o defeito ósseo ocorre por meio da pressão imposta pela glândula, o que é suportado pela idade dos pacientes que apresentam esta condição ${ }^{6}$. Devido à incerteza de sua etiologia, percebe-se a confusão na denominação dessa cavidade óssea. Outras nomenclaturas que podem ser utilizadas são:

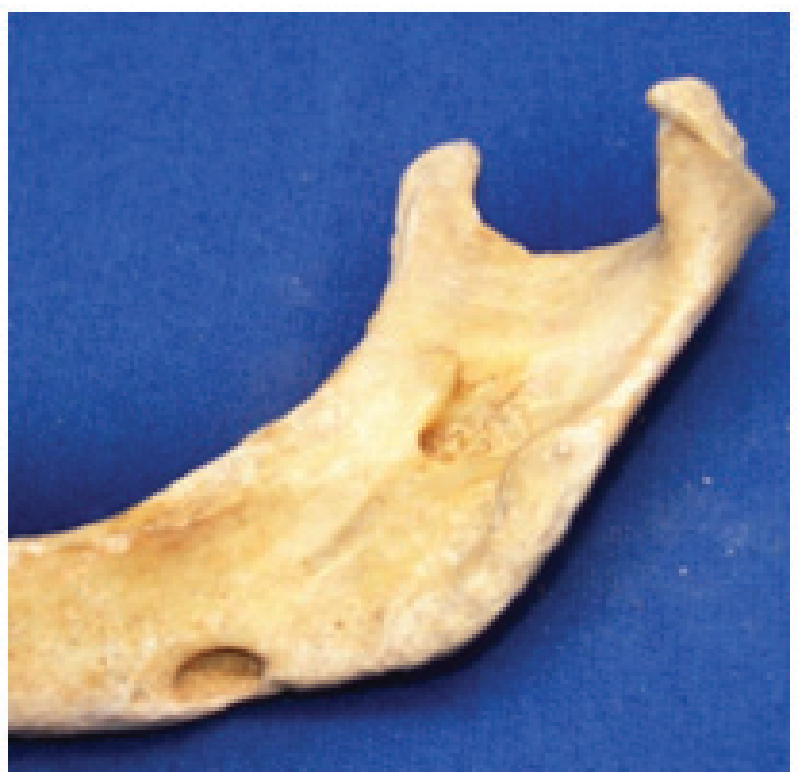

FIG. 1: CONCAVIDADE NA SUPERFÍCIE LINGUAL DA REGIÃO DO CORPO DE UMA MANDÍBULA SECA. cavidade óssea idiopática, defeito embrionário mandibular, cisto ósseo latente, defeito ósseo estático, cavidade óssea estática, defeito aberrante da glândula salivar, defeito cortical lingual mandibular e pseudocisto de Stafne ${ }^{7}$.

Essa cavidade óssea é visualizada em exames radiográficos de rotina e na grande maioria das vezes é um achado radiográfico incidental. O diagnóstico pode ser realizado por meio de radiografias convencionais, porém frequentemente pode ser necessário utilizar outros tipos de recursos de imagem, como tomografia computadorizada, sialografia ou ressonância magnética para concluir o diagnóstico, uma vez que esta condição pode ser confundida com processos patológicos ${ }^{4}$.

Exemplificando, na figura 1 pode ser observado o defeito ósseo de Stafne em uma mandíbula seca, onde visualiza-se a presença de uma concavidade localizada na superfície lingual da região posterior do corpo da mandíbula do lado direito. $\mathrm{Na}$ imagem radiográfica periapical da mesma área (figura 2) verifica-se uma imagem radiolúcida unilocular, bem delimitada por um halo radiopaco, exceto no limite inferior, e localizada abaixo do canal mandibular.

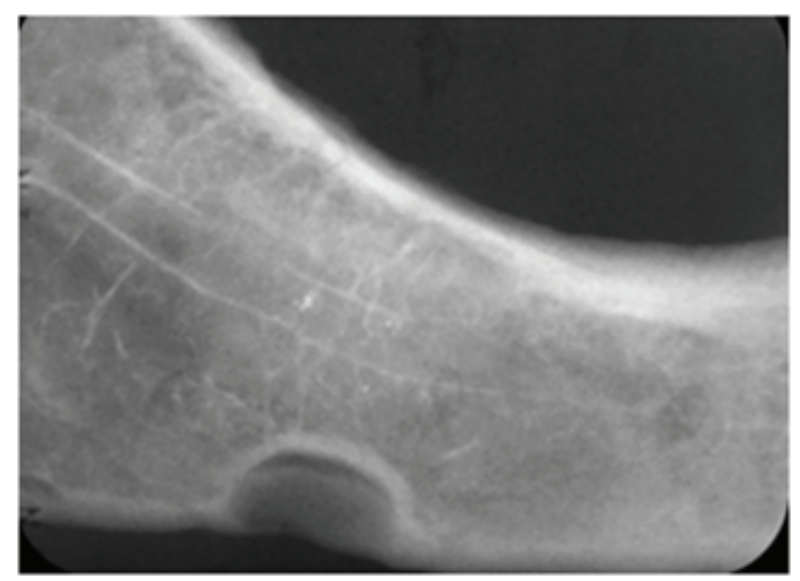

FIG. 2: IMAGEM RADIOGRÁFICA A MANDÍBULA SECA, MOSTRANDO AS CARACTERÍSTICAS RADIOGRÁFICAS DO DEFEITO ÓSSEO DE STAFNE. 


\section{Caso Clínico}

O relato desse caso foi elaborado a partir das imagens tomográficas obtidas em 2009, que pertencem ao arquivo da Clínica Odonto Radiológica Spyro Spyrides, cujo responsável autorizou a utilização dessas imagens, respeitando as normas da Resolução 196/96 do Ministério da Saúde.

Paciente do sexo masculino, com 63 anos de idade, foi encaminhado a uma clínica particular na cidade do Rio de Janeiro para realização de uma tomografia computadorizada de feixe cônico (TCFC), com a finalidade de colocação de implantes dentários na região de dentes posteriores inferiores do lado esquerdo. O paciente não apresentava qualquer sintomatologia.

Para a aquisição da imagem de TCFC foi utilizado o aparelho I-CAT $®$ (Imaging Sciences International, Hatfield, Pennsylvania, EUA), operando com $120 \mathrm{Kv}, 36,12 \mathrm{~mA}$ e $40 \mathrm{seg}$. de exposição. O escaneamento foi realizado com $6,0 \mathrm{~cm}$ de colimação (FOV) e tamanho do voxel isotrópico foi de $0,25 \mathrm{~mm}$.
Na reconstrução panorâmica da TCFC foi observada uma imagem hipodensa bem delimitada por um halo hiperdenso, localizada na região posterior do corpo da mandíbula do lado esquerdo, e sobreposta ao canal mandibular adjacente. Esta imagem apresentava forma arredondada, medindo aproximadamente 10,25mm em direção ântero-posterior e 8,85mm em altura (Figura 3).

Na reconstrução axial, pode-se notar a depressão óssea na cortical lingual do corpo da mandíbula, com limites nítidos, e integridade da cortical óssea vestibular. Em virtude da localização e das características da imagem, o diagnóstico foi de defeito ósseo de Stafne (Figura 4).

Já nas reconstruções parassagitais foi possível avaliar a relação do defeito ósseo com o canal mandibular. Assim, podese constatar que a depressão óssea está localizada por lingual em relação ao canal mandibular, e íntima relação com o mesmo, porém não é observado deslocamento ou qualquer alteração dessa estrutura anatômica (Figura 5).

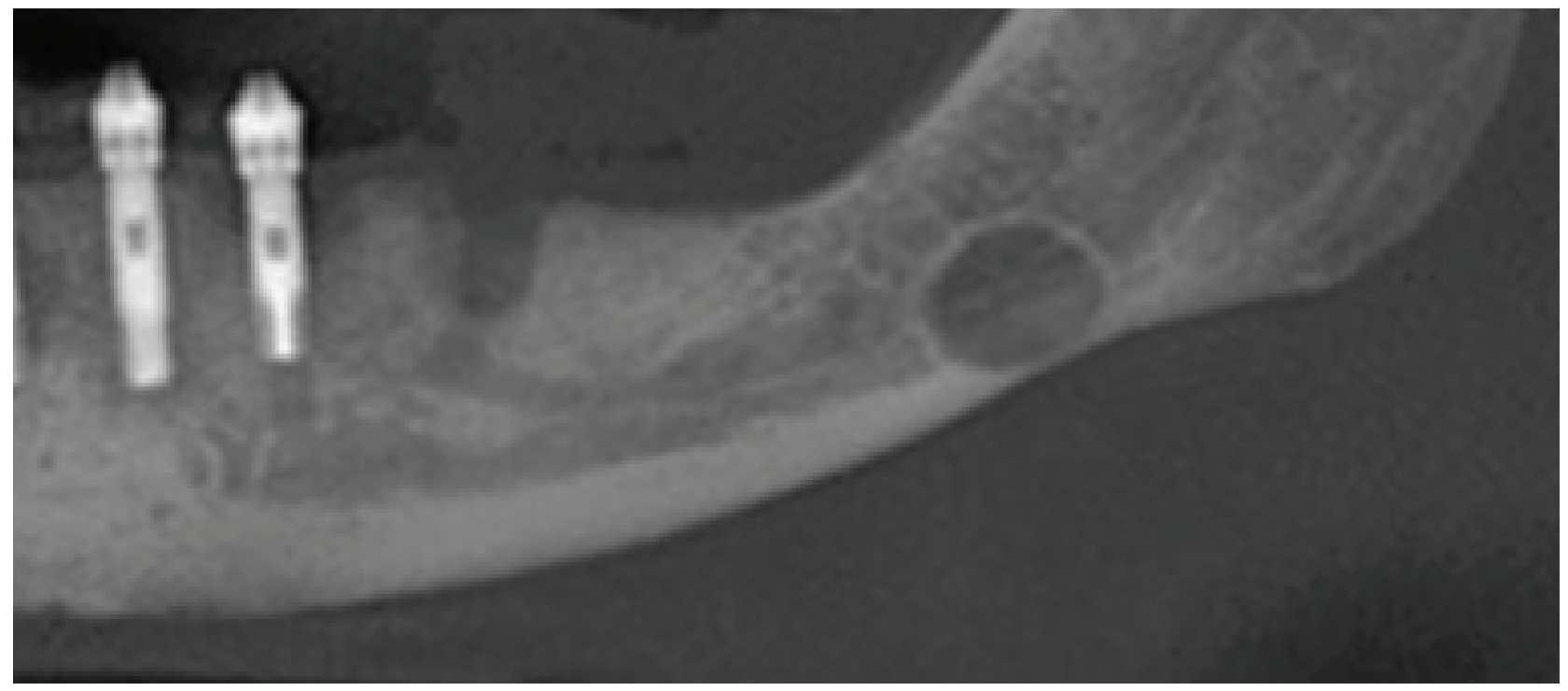

FIg. 3: IMAgEM HIPODENSA, BEM DELIMITADA POR UM HALO HIPERDENSO, LOCALIZADA NA REGIÃO POSTERIOR DO CORPO DA MANDÍBULA DO LADO ESQUERDO (RECONSTRUÇÃO PANORÂMICA DA TCFC). 


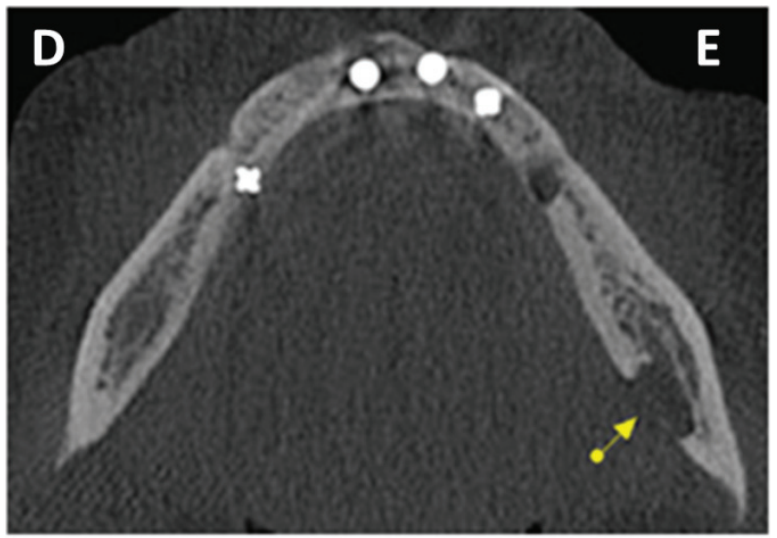

Fıg. 4: DePRESSÃo NA SUPERFÍCIE LINGUAL DA MANDíBULA EM REGIÃO DE DENTES POSTERIORES DO LADO ESQUERDO (RECONSTRUÇÃo AXIAL DA TCFC)
$\mathrm{Na}$ reconstrução tridimensional observa-se, de maneira ilustrativa, a proximidade do defeito ósseo com o canal mandibular (Figura 6).

\section{Dıscussão}

O defeito ósseo de Stafne é uma condição assintomática, com predileção por homens entre quinta e sétima décadas de vida e, na maioria das vezes, são solitárias e unilaterais ${ }^{8}$, semelhante ao que foi observado no presente relato de caso. Esta condição também pode acometer a região anterior do corpo da mandíbula, porém é mais rara e relacionada à glândula salivar sublingual ${ }^{1,4,9}$. Os defeitos ósseos que ocorrem em região anterior são

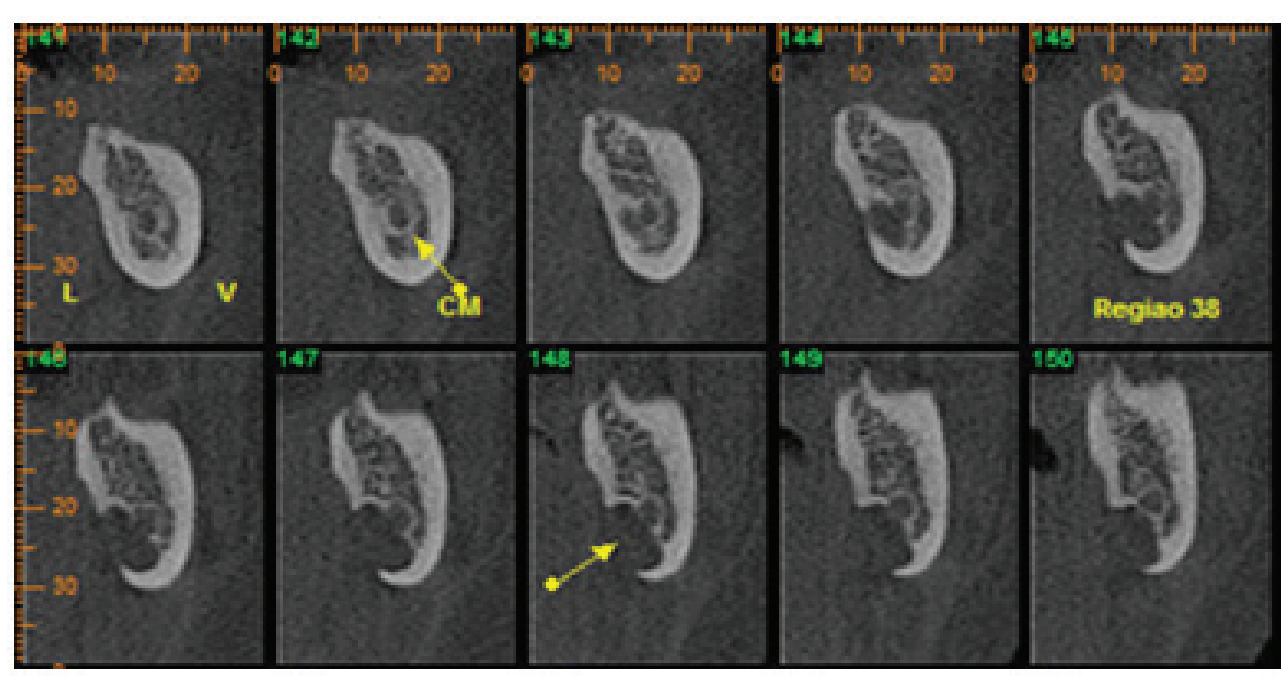

FIG. 5: RECONSTRUÇõES PARASSAGITAIS DEMONSTRAM A RELAÇÃO DA DEPRESSÃO ÓSSEA COM O CANAL MANDIBULAR ADJACENTE.

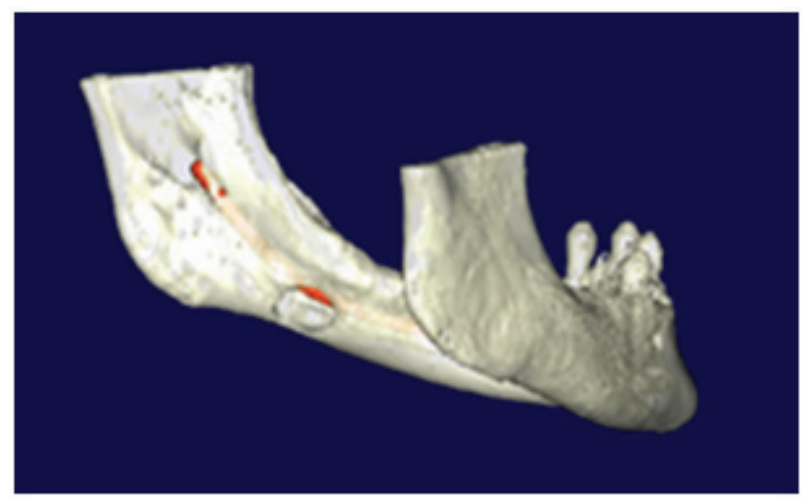

FIG. 6: RECONSTRUÇÃO TRIDIMENSIONAL PARA

VISUALIZAÇÃO DA CAVIDADE ÓSSEA NA SUPERFÍCIE LINGUAL E SUA RELAÇÃO COM O CANAL MANDIBULAR. de diagnóstico mais difícil, uma vez que não é possível utilizar como parâmetro a relação com o canal mandibular e podem se apresentar sobrepostos aos ápices radiculares, dando uma ideia de lesão cística verdadeira.

A etiologia do defeito ósseo de Stafne é incerta. A teoria mais adotada é que a cavidade se desenvolva devido uma pressão contínua das estruturas adjacentes, como a glândula salivar submandibular, formando a concavidade ${ }^{1,3,4}$. No entanto, existem aqueles que acreditam que a cavidade representa um defeito congênito, ou é causada por atrofia localizada no osso, sendo resultado de uma isquemia ${ }^{10}$. A glândula salivar submandibular não é a única causa do defeito e, tampouco, o único tecido encontrado, porém as cavidades de maior extensão e que afetam a cortical vestibular são as que possuem o tecido da glândula salivar submandibular ${ }^{7,10}$. 
Essas cavidades são normalmente visualizadas em exames radiográficos de rotina, como uma imagem radiolúcida, unilocular, bem delimitada, em região posterior do corpo da mandíbula, particularmente próxima ao ângulo da mandíbula e abaixo do canal mandibular. ${ }^{1,2,3,5}$ Características semelhantes foram visualizadas no relato de caso em questão.

As radiografias convencionais, complementares à história clínica do paciente, podem não ser suficientes para o diagnóstico, especialmente quando se trata de uma lesão atípica, com margem esclerótica incompleta, bilateral, ou localização incomum, podendo apresentar semelhança com outras condições patológicas. Devido às características da sua imagem radiográfica, os diagnósticos diferenciais do defeito ósseo de Stafne incluem lesões benignas e malignas dos maxilares, como: lesões císticas odontogênicas (cisto residual, cisto radicular), displasia fibrosa, lesões vasculares, tumor marrom do hiperparatireoidismo, síndrome nevóide basocelular, tumor de células gigantes, metástase de um tumor maligno primário ${ }^{11}$, cisto ósseo aneurismático, ameloblastoma unicístico e tumor queratocístico odontogênico ${ }^{8}$.

Segundo Dorman \& Pierse (2002), em estudo utilizando imagens de radiografia panorâmica, o diagnóstico correto do defeito ósseo de Stafne só poderia ser dado após exploração cirúrgica e exame histológico ${ }^{12}$. Perante a dificuldade de se realizar o diagnóstico preciso dessa imagem por meio de radiografias, os exames de ressonância magnética, tomografia computadorizada e tomografia computadorizada de feixe cônico passaram a ser indicados para melhor avaliação, já que permitem a visualização da terceira dimensão, além do exame de sialografia.

Segundo alguns autores, as tomografias computadorizadas devem ser o exame de primeira escolha para o diagnóstico do defeito ósseo de Stafne quando o conteúdo não precisa ser investigado, e quando a localização e as imagens características não podem ser vistas nos exames radiográficos convencionais ${ }^{4}$. De acordo com Caputo et al. (2009), a TCFC mostrou relevante importância para o diagnóstico do defeito ósseo lingual da mandíbula, ao permitir a visualização da relação deste com a cortical óssea e sua etiologia, além de utilizar menor dose de radiação, em relação à TC médica, e alto detalhamento da imagem ${ }^{13}$. Ariji et al. (1993), classificaram o defeito ósseo de Stafne, em cortes sagitais de tomografia computadorizada, de acordo com a profundidade da concavidade e tecido mole presente. Segundo os autores a visualização da concavidade lingual é um aspecto de grande importância para a conclusão do diagnóstico, imagem essa que pode ser visualizada por meio de TCFC ${ }^{10}$.

Somando-seapresençadaconcavidade lingual visualizada por cortes sagitais e axiais de TCFC, às demais características radiográficas do defeito ósseo de Stafne descrita por alguns autores, observa-se a semelhança destes relatos encontrados na literatura com o que foi observado no presente relato de caso.

A ressonância magnética deve ser utilizada apenas quando as tomografias computadorizadas e radiografias convencionais forem incapazes de dar um preciso diagnóstico, sendo que a grande vantagem deste exame é a identificação precisa do tecido mole, que está presente na cavidade, sem a utilização da radiação ionizante ${ }^{4}$.

A sialografia, exame radiográfico em que se utiliza a introdução de meios de contraste no interior do sistema de ductos das glândulas salivares, pode ser importante para a confirmação do diagnóstico do defeito ósseo de Stafne, porém devemos lembrar que este é um exame invasivo, podendo ser incômodo ao paciente. Segundo alguns autores este exame é contraindicado nos casos associados à 
glândula salivar sublingual, devido à presença de numerosos ductos de Rivini que possuem menor diâmetro, podendo ocasionar trauma e ruptura ${ }^{1,8,14}$. Este exame também não deve ser realizado quando o paciente relata possuir alergia ao meio de contraste.

A indicação de um correto exame de imagem é de grande importância a fim de se evitar biópsia ou exploração cirúrgica desnecessária, já que por se tratar de uma variação anatômica, não é necessário o tratamento do defeito ósseo de Stafne.

\section{Conclusão}

Observou-se, neste relato de caso, que a TCFC é um exame complementar indicado para o diagnóstico do defeito ósseo de Stafne, pois permite a visualização da concavidade presente na cortical óssea lingual, em região posterior do corpo da mandíbula, e sua localização mais inferior com relação ao canal mandibular, além de outras características radiográficas, descartando a possibilidade de outras condições patológicas.

O diagnóstico por meio da TCFC possibilita maior conforto ao paciente, empregando menor dose de radiação quando comparado a TC médica, e evita a exploração cirúrgica desnecessária da condição.

\section{REFERÊNCIAS BIBLIOGRÁFICAS}

1. de Courten A, Küffer R, Samson J, et al. Anterior lingual mandibular salivary gland defect (Stafne defect) presenting as a residual cyst. Oral Surg Oral Med Oral Pathol Oral Radiol Endod 2002;94(4):460-4.

2. Kursoglu P, Ari N, Calikkocaoglu S. Use of Stafne's mandibular defect in improving retention of mandibular complete dentures. $\mathrm{N} \mathrm{Y}$ State Dent J 2007;73(5):52-4.

3. Queiroz LM, Rocha RS, de Medeiros KB, et al. Anterior bilateral presentation of Stafne defect: an unusual case report. J Oral Maxillofac Surg 2004;62(5):613-5.

4. Sisman $Y$, Etöz OA, Mavili E, et al. Anterior
Stafne bone defect mimicking a residual cyst: a case report. Dentomaxillofac Radiol 2010;39(2):124-6.

5. Jordana X, García SC, Galtés I, et al. Report on a Stafne defect in a man from medieval age. J Oral Maxillofac Surg 2007;65(3):556-9.

6. Krafft T, Eggert J, Karl M. A Stafne bone defect in the anterior mandible--a diagnostic dilemma. Quintessence Int 2010;4(5):391-3.

7. Manzi FR, Guedes FR, Lima JJG et al. Características radiográficas e tomográficas do defeito ósseo de Stafne. Arq Bras Odontol 2005;1(1):34-43.

8. Turkoglu K, Orhan K. Stafne bone cavity in the anterior mandible. J Craniofac Surg 2010;21(6):1769-75.

9. Bornstein $M M$, Wiest $R$, Balsiger $R$, et al. Anterior Stafne's bone cavity mimicking a periapical lesion of endodontic origin: report of two cases. J Endod 2009;35(11):1598-602.

10. Ariji E, Fujiwara N, Tabata O. et al. Stafne's bone cavity: classification based on outline and content determined by computed tomography. Oral Surg Oral Med Oral Pathol 1993;76(3):375-80.

11. Segev $Y$, Puterman M, Bodner L. Stafne bone cavity--magnetic resonance imaging. Med Oral Patol Oral Cir Bucal 2006;11(4):E345-7.

12. Dorman M, Pierse D. Ectopic salivary gland tissue in the anterior mandible: a case report. $\mathrm{Br}$ Dent J 2002;193(10):571-2.

13. Caputo BV, Felicore, SM, Giovani EM, et al. Validação da tomografia computadorizada de feixe cônico como recurso no diagnóstico do defeito ósseo lingual na mandíbula. Rev Inst Ciênc Saúde 2009;27(4):422-5.

14. Smith MH, Brooks SL, Eldevik OP, et al. Anterior mandibular lingual salivary gland defect: a report of a case diagnosed with cone-beam computed tomography and magnetic resonance imaging. Oral Surg Oral Med Oral Pathol Oral Radiol Endod 2007;103(5):e71-8.

Autor para contato

Eduardo Murad Villoria

E-mail: duduvilloria@yahoo.com.br

Submetido em: 6-3-2013

Aceito em: 15-6-2013 